\title{
Time-variant reliability analysis of a continuous system with strength deterioration based on subset simulation
}

\author{
Xi-Nong En ${ }^{1} \cdot$ Yi-Min Zhang ${ }^{2} \cdot$ Xian-Zhen Huang ${ }^{1}$
}

Received: 8 November 2018/Revised: 24 December 2018/Accepted: 1 April 2019/Published online: 28 May 2019

(C) The Author(s) 2019

\begin{abstract}
To conduct a reliability analysis for mechanical components, it is necessary to consider the combined influence of strength deterioration and dynamic loads. An efficient method based on subset simulation is proposed in this paper to analyze time-variant reliability by considering the strength deterioration of mechanical components in a continuous system. A gamma process is used to describe the deterioration of system strength. A model for timevariant reliability considering strength deterioration is constructed for a continuous system. A representative example and tubular cantilever structure are assessed to demonstrate the efficiency and accuracy of the proposed method. The reliability probability examples were analyzed using a first-order reliability method and benchmark results for the proposed method were derived using direct Monte Carlo simulation (MCS). The results of the proposed method and MCS are consistent, indicating that the proposed method is an effective reliability analysis method for evaluating small failure probabilities in a continuous system subjected to strength deterioration and dynamic loads.
\end{abstract}

Yi-Min Zhang

ymzhang@mail.neu.edu.cn

Xian-Zhen Huang

xzhhuang@mail.neu.edu.cn

1 School of Mechanical Engineering and Automation, Northeastern University, Shenyang 110819, People's Republic of China

2 Equipment Reliability Institute, Shenyang University of Chemical Technology, Shenyang 110142, People's Republic of China
Keywords Time-variant reliability $\cdot$ Strength deterioration - Subset simulation (SS) · Continuous system

\section{Introduction}

The reliability analysis of mechanical components is critical for improving the operational reliability and efficiency of mechanical equipment. Considering the influence of changes in the environment and uneven material surfaces, the structural and performance parameters of mechanical components are often uncertain. Some parameters are timeinvariant random variables subjected to a particular distribution (e.g., normal distribution), while others are timevariant random variables that change regularly over time or follow a random process. According to different typologies of uncertainty, analysis methods for reliability can be divided into two main categories: time-variant and timeinvariant methods. Over the past few years, several studies on reliability methods have been reported. Such methods have been widely utilized in engineering applications [1-3]. Castaldo et al. [1] proposed a sectional approach based on Monte Carlo simulation (MCS) to estimate the expected lifetime of a deteriorating reinforced concrete bridge pier. Huang et al. [2] proposed a new second-order reliability method with saddle point approximation for accurate, convergent, and computationally efficient estimates of the probability of failure. Zhu et al. [3] incorporated finite element simulations with Latin hypercube sampling to assess the fatigue reliability of high-pressure turbine discs under complex loadings coupled with multisource uncertainties.

In most cases, mechanical components are subjected to strength deterioration and the failure process is gradual. Several methods have been reported for analyzing 
component deterioration. Mori and Ellingwood [4] developed methods to evaluate the time-dependent reliability of reinforced or pre-stressed concrete structures using structural reliability principles. Li [5] postulated that the timedependent reliability analysis of a deteriorating structural system was based on the reliability of a given failure sequence for the structure. Ciampoli [6] formulated a probabilistic method based on a stochastic differential equation for the reliability assessment of structural components subjected to deterioration. Li et al. [7] developed an improved method for evaluating the time-dependent reliability of structures to assess the effects of nonstationarity in the load and resistance deterioration processes on safety and serviceability quantitatively.

Mechanical components typically bear dynamic loads during regular system operation. Therefore, it is essential to evaluate the continuous reliability of mechanical components by combining dynamic loads with strength deterioration. Rice [8] presented a novel first-passage probability formula, which has been widely used to evaluate timevariant reliability. Andrieu-Renaud et al. [9] developed a method known as PHI2 based on an out-crossing approach that solved reliability problems using classical time-invariant reliability tools, such as the first-order reliability method (FORM) and second-order reliability method. Zhang et al. [10] proposed an approach to translate timevariant reliability into static reliability by discretizing the stochastic processes of resistance and stress at calculation time. Li and Kiureghian [11] developed a method based on the principles of optimal linear estimation theory for the efficient discretization of random fields to simulate stochastic processes.

Subset simulation (SS) was originally proposed by $\mathrm{Au}$ and Beck [12] to estimate the small failure probability of high-dimensional parameter space problems. Small failure probability is expressed as the product of the large conditional probabilities of selected intermediate failure events. This method has been widely used in statistics, applied mathematics, and structural engineering. Au and Beck [13] used SS to effectively simulate procedure for seismic performance assessment of structures in the context of modern performance-based design. Vahdatirad et al. [14] estimated low-event probabilities using SS to analyze the first natural frequency of a turbine supported by a surface footing. Norouzi and Nikolaidis [15] re-evaluated the reliability of a dynamic system subjected to stationary Gaussian stochastic excitation for various load spectra by reweighting the results of a single SS. Song et al. [16] analyzed the reliability sensitivity of failure probabilities by transforming the distribution parameters of basic variables into a set of conditional failure probabilities using SS. Bourinet et al. [17] presented an approach referred as 2SMART for estimating small failure probabilities by considering SS from the perspective of support vector machine classification. Zuev et al. [18] presented a modified Metropolis algorithm based on Markov chain MCS and used SS for sampling from conditional distributions to compute small failure probabilities in general high-dimensional reliability problems.

Recently, SS has been widely used to evaluate dynamic structural reliability [19-22]. Li et al. [19] developed a generalized SS approach to estimate the failure probabilities of multiple stochastic responses. Wang et al. [20] presented an improved SS method with a splitting approach to estimate the time-dependent reliability of systems with random parameters excited by stochastic processes. Yu and Wang [21] proposed a novel time-variant reliability analysis method using failure process decomposition to transform time-variant reliability problems into time-invariant problems for dynamic structures with uncertainty. Yu et al. [22] proposed a novel time-variant reliability analysis method for multiple failure modes and temporal parameters based on a combination of the extreme value moment method and improved maximum entropy method. However, time-variant reliability problems with strength deterioration for mechanical components have rarely been evaluated using SS. In this paper, a novel approach is proposed for the continuous system reliability analysis of mechanical components using SS. Expressions for the performance functions of a continuous system are constructed using a model for time-variant reliability with strength deterioration and the failure probabilities of mechanical components are derived.

The remainder of this paper is organized as follows. Section 2 describes a model for deterioration processes and presents a performance function for a continuous system, as well as a model of time-variant reliability with strength deterioration. Section 3 describes an approach for evaluating continuous system reliability using SS. Section 4 presents two numerical examples to demonstrate the efficiency and accuracy of the proposed approach. Conclusions are provided in Section 5.

\section{Model of time-variant reliability with strength deterioration}

The gamma process proposed by Abdel-Hameed [23] is the most appropriate process for describing the deterioration of strength. Excluding cyclic loads, a given system is largely influenced by random environmental factors, such as random vibrations in the external environment, stochastic variation in ambient temperature, or corrosion. These factors lead to a monotonic decrease in strength and stochastic process evolution over time. Furthermore, the gamma process is a continuous-time stochastic process with 
independent, nonnegative increments (e.g., variation in tubular cantilever structural strength). Therefore, it is suitable for modeling gradual damage that accumulates monotonically over time in a sequence of small increments, such as wear, fatigue, corrosion, creep, and a degrading health index. Additionally, relatively straightforward mathematical calculations are a major advantage of modeling deterioration processes as gamma processes.

Van Noortwijk [24] described the statistical properties of gamma processes as a deterioration model. The symbol $Y(t)$ is used to denote the deterioration at a time $t$, where $t \geq 0$. Therefore, according to the definition of a nonstationary gamma process, the probability density function (PDF) of $Y(t)$ can be expressed as

$$
\begin{aligned}
f_{Y(t)}(y) & =G(y \mid v(t), u) \\
& =\frac{1}{\Gamma(v(t)) u^{v(t)}} y^{v(t)-1} \exp (-y / u) I_{(0, \infty)}(y),
\end{aligned}
$$

where $I_{(0, \infty)}(y)=1$ for $y \in(0, \infty), I_{(0, \infty)}(y)=0$ for $y$ $(0, \infty)$, and $\Gamma(v)=\int_{0}^{\infty} y^{v-1} \mathrm{e}^{-y} \mathrm{~d} y$ is the gamma function for a positive shape parameter $v$. Furthermore, the gamma process is a stochastic process with a shape function $v(t)$, which is non-decreasing and right-continuous, and positive scale parameter $u$. Therefore, the expected value can be expressed as

$E(Y(t))=\int_{0}^{\infty} y f_{Y(t)}(y) \mathrm{d} y=u v(t)$.

The variance can be written as

$$
\begin{aligned}
\operatorname{var}(Y(t)) & =\int_{0}^{\infty}(y-E(Y(t)))^{2} f_{Y(t)}(y) \mathrm{d} y \\
& =u^{2} v(t) .
\end{aligned}
$$

Experimental studies have confirmed that the expectation of deterioration at a time $t$ is generally proportional to the following power law

$$
\begin{aligned}
E(Y(t)) & =u c t^{b} \\
& =a t^{b} \propto t^{b},
\end{aligned}
$$

where $a, b$, and $c$ are positive physical constants.

For applying the gamma process model to engineering problems, statistical methods are required to estimate the parameters of the shape function $v(t)=c t^{b}$ and scale parameter $u$. Through various calculations, practical knowledge [25-28] can be obtained regarding the shape function in terms of the parameter $b$ in Eq.(4). Consequently, the value of $b$ is assumed to be known, but the values of $c$ and $u$ are unknown. The estimators of $c$ and $u$ can be obtained using the most common method of parameter estimation, namely the maximum likelihood.
Cinlar et al. [26] provided a method for transforming a nonstationary gamma process into a stationary gamma process. The corresponding observations of cumulative deterioration $\quad\left\{y_{i_{\mathrm{t}}} \mid i_{\mathrm{t}}=1,2, \cdots, n_{\mathrm{t}}\right\}, \quad$ where $y_{0}<y_{1}<y_{2}<\cdots<y_{n_{\mathrm{t}}}$ and $y_{0}=0$, are obtained at inspection time points $\left\{t_{i_{\mathrm{t}}} \mid i_{\mathrm{t}}=1,2, \cdots, n_{\mathrm{t}}\right\}$, where $t_{0}<t_{1}<t_{2}<\cdots<t_{n_{\mathrm{t}}}$ and $t_{0}=0$. The likelihood function for the observed deterioration increments $\left\{\delta_{i_{\mathrm{t}}}=y_{i_{\mathrm{t}}}-y_{i_{\mathrm{t}}-1} \mid i_{\mathrm{t}}=1,2, \cdots, n_{\mathrm{t}}\right\}$ is used to estimate the values of $\hat{c}$ and $\hat{u}$.

Based on the above analysis, the residual strength $S(t)$, when formulated as a stochastic process, is equal to the initial strength $S_{0}$, and is assumed to decrease monotonically based on a deterioration function $Y(t)$. Therefore, the general form of assumed residual strength can be expressed as

$S(t)=S_{0}-Y(t)$,

where $S_{0}$ is a random variable with a normal distribution, which is closely related to component elements, internal defects, heat treatment, and work-hardening of materials. $Y(t)$ is a stochastic process depending on the conditions of suffered stress and operating environment. Therefore, $S_{0}$ and $Y(t)$ are uncorrelated.

Based on the above definitions and the stress-strength interference theory, the performance function for timevariant reliability with strength deterioration for a continuous system can be expressed as

$g(\boldsymbol{X}, t)=S(t)-\max (\sigma(\boldsymbol{Z}, t))$,

where the stochastic process $\boldsymbol{X}(t)$ is composed of the initial strength $S_{0}$, deterioration function $Y(t)$, and external loads $\boldsymbol{Z}(t)$ which include the static and dynamic loads at the time $t$. Therefore, $\max (\sigma(\boldsymbol{Z}, t))$ denotes the maximum dynamic stress magnitude imposed by external loading during the time interval.

Consequently, the failure probability of a continuous system with strength deterioration can be calculated as

$$
\begin{aligned}
P_{\mathrm{F}}(t) & =P\{g(\boldsymbol{X}, t)<0\} \\
& =P\{S(t)-\max (\sigma(\boldsymbol{Z}, t))<0\} .
\end{aligned}
$$

The model of time-variant reliability can be expressed as

$R(t)=1-P_{\mathrm{F}}(t)$.

MCS is generally a feasible method to estimate the probability of failure once a system performance function is formulated. However, MCS is not appropriate for obtaining small probabilities of failure (e.g., $P_{\mathrm{F}}<10^{-3}$ ) because it suffers from a crucial lack of efficiency to achieve the required accuracy. Therefore, in this study, a Markov chain Monte Carlo (MCMC) method based on the Metropolis algorithm was used to efficiently compute small failure probabilities in a continuous system bearing 
dynamic loads and suffering from gradual failure caused by strength deterioration.

\section{SS-based time-variant reliability analysis with strength deterioration}

The core idea of the SS method proposed by Au and Beck [12] is to estimate the frequency of a rare event based on the frequencies of more common events in a sequence of intermediate failure events. SS has been widely used to analyze reliability effectively. However, solving the problem of time-variant reliability analysis with strength deterioration using SS has received little attention. Based on the performance function described in Section 2, SS is expanded in this section to handle the problem of timevariant reliability analysis with strength deterioration for a continuous system.

\subsection{SS for a continuous system}

Regardless of whether or not the stochastic process $\boldsymbol{X}(t)=$ $\left(S_{0}, Y(t), \boldsymbol{Z}(t)\right)$ is defined with respect to continuous-time or discrete-time variables, only the values for a process at a discrete number of time instances are generated. For the sake of convenient calculation, this time grid is defined by $0, t_{1}, t_{2}, \cdots, t_{n_{\mathrm{t}}-1}, t_{n_{\mathrm{t}}}$, where $t_{i_{\mathrm{t}}}=\left(i_{\mathrm{t}} / n_{\mathrm{t}}\right) t$ for $t>0$ and $i_{\mathrm{t}}=0,1, \cdots, n$. Then, $\boldsymbol{X}(t)$ can be expressed as vectors of random variables $\boldsymbol{X}_{1}, \boldsymbol{X}_{2}, \cdots, \boldsymbol{X}_{n_{\mathrm{t}}-1}, \boldsymbol{X}_{n_{\mathrm{t}}}$ (i.e., $\left\{\boldsymbol{X}_{i_{\mathrm{t}}} \mid i_{\mathrm{t}}=1\right.$, $\left.2, \cdots, n_{\mathrm{t}}\right\}$ ).

The symbols $\left\{F_{i_{\mathrm{t}}} \mid i_{\mathrm{t}}=1,2, \cdots, n_{\mathrm{t}}\right\}$ define the continuous system failure events at the time instances $t_{i_{\mathrm{t}}}$. Furthermore, $F_{i_{\mathrm{t}}, i}=\left\{g\left(\boldsymbol{X}_{i_{\mathrm{t}}}\right)<b_{i}\right\}, i=1,2, \cdots, m$, where $F_{i_{\mathrm{t}}, 1} \supset F_{i_{\mathrm{t}}, 2} \supset$ $\cdots \supset F_{i_{t}, m}=F_{i_{\mathrm{t}}}$, denotes a set of intermediate conditional failure events with a decreasing sequence of intermediate threshold values $b_{1}>b_{2}>\cdots>b_{m-1}>b_{m}=0$. Consequently, the probability of failure at a time $t_{t_{\mathrm{t}}}$ can be expressed as

$$
\begin{aligned}
P_{\mathrm{F}, i_{\mathrm{t}}} & =P\left(g\left(\boldsymbol{X}_{i_{\mathrm{t}}}\right)<0\right)=P\left(F_{i_{\mathrm{t}}}\right)=P\left(F_{i_{\mathrm{t}}, m}\right) \\
& =P\left(F_{i_{\mathrm{t}}, m} \mid F_{i_{\mathrm{t}}, m-1}\right) P\left(F_{i_{\mathrm{t}}, m-1}\right)=\cdots \\
& =P\left(F_{i_{\mathrm{t}}, 1}\right) \prod_{i=2}^{m} P\left(F_{i_{\mathrm{t}}, i} \mid F_{i_{\mathrm{t}}, i-1}\right),
\end{aligned}
$$

where $\boldsymbol{X}_{i_{\mathrm{t}}}=\left[X_{i_{\mathrm{t}}, 1}, X_{i_{\mathrm{t}}, 2}, \cdots, X_{i_{\mathrm{t}}, n}\right]$ is a vector of random variables with a $\operatorname{PDF} q\left(\boldsymbol{X}_{i_{\mathrm{t}}}\right)=\prod_{j=1}^{n} q_{j}\left(\boldsymbol{X}_{i_{\mathrm{t}}}(j)\right)$, where $n$ is the number of variables. The symbol $q_{j}(\cdot)$ represents the univariate PDF for each component of $\boldsymbol{X}_{i_{t}}$.

Equation (9) calculates the product of a sequence of intermediate conditional probabilities $\left\{P\left(F_{i_{t}, i} \mid F_{i_{t}, i-1}\right) \mid i=\right.$ $2,3, \cdots, m\}$ and the first level $P\left(F_{i_{t}, 1}\right)$. To make the conditional probabilities in Eq.(9) sufficiently large to be estimated efficiently, it is important to select intermediate failure events appropriately. Particularly, the prior determination of $b_{i}$ is not a trivial task. Therefore, in this study, $b_{i}$ was selected such that the estimated conditional probabilities were equal to a fixed value $p_{0} \in(0,1)$.

This was accomplished by setting the intermediate threshold values $\left\{b_{i} \mid i=1,2, \cdots, m-1\right\}$ equal to the $\left(p_{0} N_{1}+1\right)$ th values of $\left\{g\left(\boldsymbol{X}_{i_{1}, k_{1}}^{(i-1)}\right) \mid k_{1}=1,2, \cdots, N_{1}\right\}$, sorted in an ascending order. The symbols $\left\{\boldsymbol{X}_{i_{\mathrm{t}}, k_{1}}^{(i-1)} \mid i=2,3, \cdots, m-1\right\}$ denote the samples generated at the $(i-1)$ th conditional level. $\boldsymbol{X}_{i_{t}, k_{1}}^{(0)}$ are the independent and identically distributed (i.i.d.) samples simulated by the original MCS according to the PDF $q\left(\boldsymbol{X}_{i_{1}, k_{1}}^{(0)}\right) . N_{1}$ is the number of samples at each level. In this study, the value of $p_{0}$ was set to 0.1 for the sake of efficiency.

Additionally, the probabilities $P\left(F_{i_{t}, 1}\right)$ and $\left\{P\left(F_{i_{t}, i} \mid F_{i_{t}, i-1}\right) \mid i=2,3, \cdots, m\right\}$ are required to obtain $P_{\mathrm{F}, t_{t_{t}}}$ from Eq.(9). MCS can be used to estimate $P\left(F_{i_{t}, 1}\right)$ as

$P\left(F_{i_{t}, 1}\right)=\frac{1}{N_{1}} \sum_{k_{1}=1}^{N_{1}} I_{F_{i_{t}, 1}}\left(\boldsymbol{X}_{i_{\mathrm{t}}, k_{1}}^{(0)}\right)$,

where $I_{F_{i, 1}}(\cdot)$ is an indicator function. If $\boldsymbol{X}_{i_{t}, k_{1}}^{(0)} \in F_{i_{t}, 1}, I_{F_{i_{t}, 1}}\left(\boldsymbol{X}_{i_{t}, k_{1}}^{(0)}\right)=1$. Otherwise $I_{F_{i_{t}, 1}}\left(\boldsymbol{X}_{i_{t}, k_{1}}^{(0)}\right)=0$. Similarly, $P\left(F_{i_{t}, i} \mid F_{i_{t}, i-1}\right)$ can be computed using a formula similar to Eq.(10). However, to obtain samples according to the conditional distributions $q\left(\boldsymbol{X}_{i_{\mathrm{t}}} \mid F_{i_{\mathrm{t}}, i}\right)=q\left(\boldsymbol{X}_{i_{\mathrm{t}}}\right) I_{F_{i_{t}, i}}$ $\left(\boldsymbol{X}_{i_{t}, k_{1}}\right) / P\left(F_{i_{i}, i}\right)$ efficiently, simulation should be carried out using Markov chain MCS based on the modified Metropolis algorithm proposed by Papaioannou et al. [29], rather than traditional MCS.

\subsection{Implementation procedure}

In general, SS for a continuous system involves six main steps, which are detailed below.

(i) Discretize the duration of continuous system operation $T$ using an appropriate sampling interval $\Delta t$ to obtain a number of time instances $n_{\mathrm{t}}=T / \Delta t$ and discrete time instances $\left\{t_{i_{\mathrm{t}}}=i_{\mathrm{t}} \Delta t \mid i_{\mathrm{t}}=0,1, \cdots, n_{\mathrm{t}}\right\}$.

(ii) At time instance $t_{1}$, generate $N_{1}$ i.i.d. samples $\boldsymbol{X}_{1}^{(0)}$ with a size of $n$ using MCS as the first level of SS.

(iii) Substitute these $N_{1}$ samples into the performance function and sort $\left\{g\left(\boldsymbol{X}_{1, k_{1}}^{(0)}\right) \mid k_{1}=1,2, \cdots, N_{1}\right\}$ in ascending order. To make $P\left(F_{1,1}\right)=$ 
$P\left\{g\left(X_{1}\right)<b_{1}\right\}$ equal to $p_{0}$, assign the $\left(p_{0} N_{1}+1\right)$ th value to $b_{1}$.

(iv) For every $i=2,3, \cdots$, generate $\left(1-p_{0}\right) N_{1}$ extra conditional samples based on the $p_{0} N_{1}$ seed samples in the failure region $F_{1, i-1}=$ $\left\{g\left(\boldsymbol{X}_{1}\right)<b_{i-1}\right\}$ using the modified Metropolis algorithm $[29,30]$ to obtain a total of $N_{1}$ samples (i.e., $\left.\boldsymbol{X}_{1, k_{1}}^{(i-1)}\right)$ in $F_{1, i-1}=\left\{g\left(\boldsymbol{X}_{1}\right)<b_{i-1}\right\}$. Similarly, calculate the values of the performance function using these $N_{1}$ samples and sort $\left\{g\left(\boldsymbol{X}_{1, k_{1}}^{(i-1)}\right) \mid k_{1}=1,2, \cdots, N_{1}\right\}$ in ascending order again. Assuming that $P\left(F_{i} \mid F_{i-1}\right)=$ $P\left\{g\left(\boldsymbol{X}_{1}\right)<b_{i-1} \mid g\left(\boldsymbol{X}_{1}\right)<b_{i}\right\}$ are equal to $p_{0}$, take the $\left(p_{0} N_{1}+1\right)$ th values of the sequence as $b_{i}$ to obtain $F_{1, i}=\left\{g\left(\boldsymbol{X}_{1}\right)<b_{i}\right\}$. If $b_{i} \leqslant 0$, define the final level failure region as $F_{1, m}=\left\{g\left(\boldsymbol{X}_{1}\right)<b_{m}\right\}$, where $m=i$ and $b_{m}=0$.

(v) Let $N_{\mathrm{f}}$ denote the number of samples at the final level of SS. Then, the conditional probability of the final level can be calculated as

$P\left(F_{1, m} \mid F_{1, m-1}\right)=N_{\mathrm{f}} / N_{\mathrm{l}}$.

Substitute $P\left(F_{1,1}\right)=p_{0},\left\{P\left(F_{1, i} \mid F_{1, i-1}\right)=p_{0} \mid i=\right.$ $2,3, \cdots, m-1\}$, and Eq.(11) into Eq.(9), the failure probability at time instance $t_{1}$ can be denoted as

$P_{\mathrm{F}, t_{1}}=p_{0}^{m-1} \frac{N_{\mathrm{f}}}{N_{\mathrm{l}}}$.

(vi) Repeat steps (ii)-(v) for the time instances $t_{i_{\mathrm{t}}}, i_{\mathrm{t}}=2,3, \cdots, n_{\mathrm{t}}$. Then, the continuous system failure probability can be denoted as $P_{\mathrm{F}}=\left\{P_{\mathrm{F}, i_{\mathrm{t}}} \mid i_{\mathrm{t}}=1,2, \cdots, n_{\mathrm{t}}\right\}$.

In this study, SS was used to calculate the time-variant reliability for a continuous system with strength deterioration. The small failure probability is transformed into the product of larger probabilities, thereby achieving a reduction in the number of required calculations and improving calculation efficiency while maintaining high precision. Figure 1 presents the implementation process of the proposed method concisely.

\section{Example applications}

In this section, two representative examples of a high-dimensional stochastic problem and continuous engineering system are used to demonstrate the accuracy and efficiency of the proposed method. MCS is the most accurate method for solving the problem of time-variant reliability analysis. Considering the nonlinearity of the performance function, the FORM [31] was adopted based on its excellent performance and simplicity. The results obtained using the proposed method were compared with those obtained using the FORM and MCS. To achieve the desired accuracy, MCS requires $10^{k+2}-10^{k+3}$ samples if the failure probability is on the order of $10^{-k}$ [32]. The variance values for the random parameters can be obtained through reliability testing or statistical analysis of experimental data, or can be estimated based on the following principles if there is no relevant data. The variance can be estimated based on a variation coefficient. In general, for mechanical performance parameters related to metal materials, this coefficient is set to 0.05 [33]. If the system is subjected to the influence of a large number of independent factors (no dominant factor), random variables commonly follow a normal distribution [34].

\subsection{High-dimensional example}

This example was chosen to assess the performance of the proposed method for handling relatively high numbers of random variables. It is known that increasing the dimensionality of the random variable space introduces various challenges (e.g., curse of dimensionality [35]). We propose analyzing the academic example of one failure mode of a bevel gear, where $z_{12}$ is the diameter of the small gear and $z_{13}$ is the length of the contact line at the midpoint of the tooth surface. $z_{i}(i=1,2, \cdots, 11,14, \cdots, 17)$ is the coefficient of the bevel gear and $F$ is the nominal tangential force on a reference circle at the midpoint of the tooth width. The statistical distributions and parameters for the random variables are listed in Table 1. According to international standards [36], the contact stress of a bevel gear $\sigma(\boldsymbol{Z}, t)$ is expressed by the load on the bevel gear transmission. The deterioration process for the contact fatigue strength of the tooth surface $S(t)$ can be described by a gamma process. The performance function is written as

$$
\begin{aligned}
g(\boldsymbol{X}, t) & =g\left(S_{0}, Y, F, z_{1}, \cdots, z_{17}, t\right) \\
& =S(t)-\max (\sigma(\boldsymbol{Z}, t)) \\
& =\left(S_{0}-Y(t)\right) \prod_{i=1}^{6} z_{i}-\prod_{i=7}^{11} z_{i} \sqrt{\frac{F(t)}{z_{12} z_{13}} \frac{u+1}{u} \prod_{i=14}^{17} z_{i},}
\end{aligned}
$$

where $\left\{z_{i} \mid i=1,2, \cdots, 17\right\}$, and $F$ and $S_{0}$ are all independent. Here, $u=1.611$ 1. Based on the $S-N$ curves, the parameters for the deterioration process of system strength are $u=1.3822 \times 10^{-8}, b=0.05$, and $c=4.9751 \times 10^{10}$.

According to the performance function, the failure probabilities of the continuous system $P_{\mathrm{F}}$ were evaluated using the proposed method, FORM, and MCS. The results 


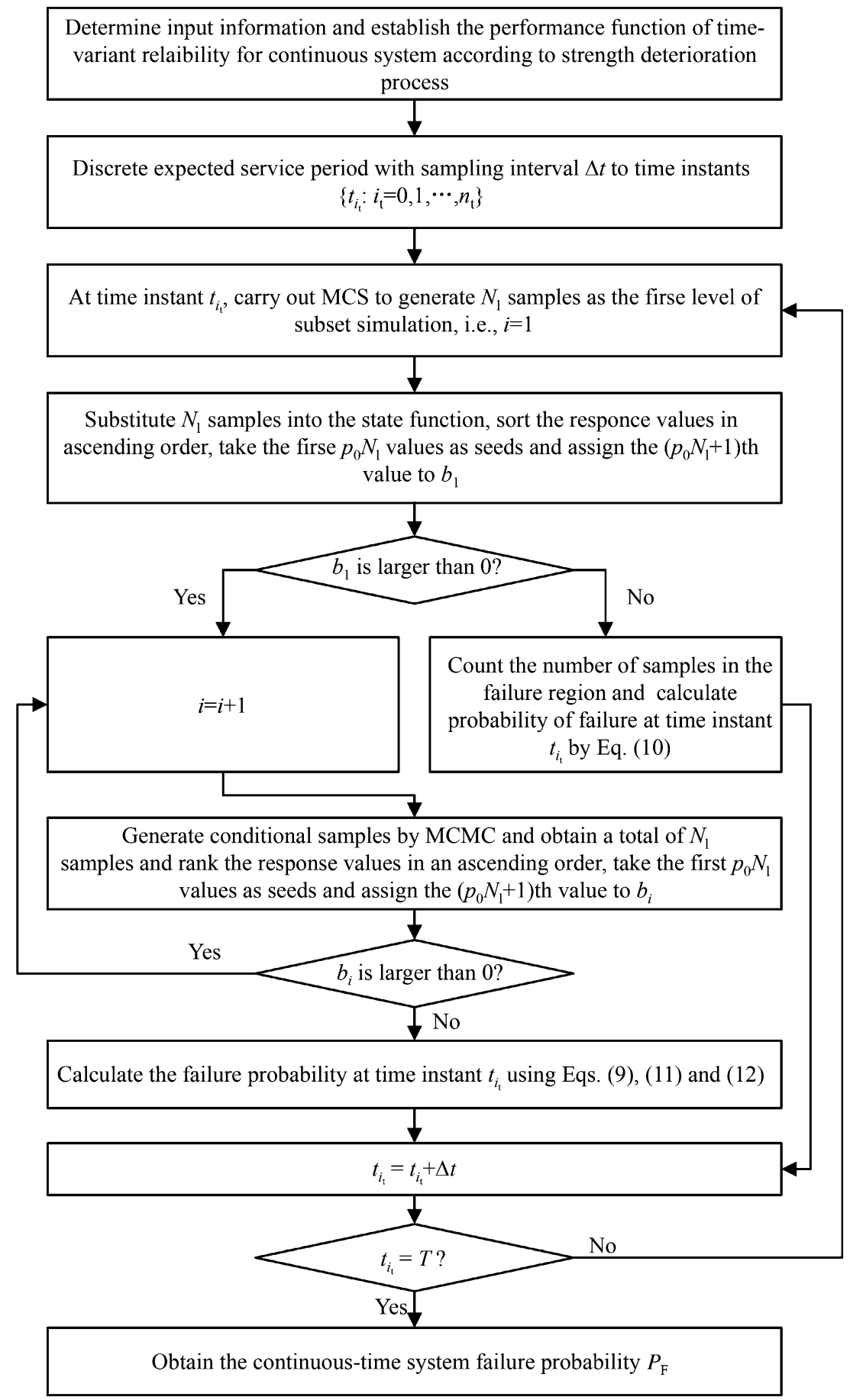

Fig. 1 Flow chart for the analysis method for the time-variant reliability of continuous systems with strength deterioration based on SS

are listed in Table 2. Figure 2 presents the cumulative probability distributions of the continuous system as calculated by the proposed method and MCS at each time instance on logarithmic scales. One can see a clear connection between the cumulative probability, value of the performance function, and time of service. As shown in Table 2 and Fig. 2, the failure probabilities and cumulative probabilities of the continuous system estimated by the proposed method agree well with those acquired from MCS. Compared to the results of the proposed method, the failure probabilities for the continuous system obtained by the FORM are significantly higher or lower than those evaluated by MCS.

To illustrate the high efficiency of the proposed method relative to MCS, Figs. 3 and 4 compare the coefficient of variation $(\mathrm{COV})$ of the failure probabilities $\delta_{i_{\mathrm{t}}}[12,37]$ and 
Table 1 Probability distributions of the basic random variables

\begin{tabular}{|c|c|c|c|c|}
\hline Random variable & Distribution & Mean & Standard deviation & Autocorrelation coefficient function \\
\hline$S_{0} /\left(\mathrm{N} \cdot \mathrm{mm}^{-2}\right)$ & Normal & 1350 & 162 & - \\
\hline$F(t) / \mathrm{N}$ & Gaussian process & 1072.61 & 105.59 & $\exp \left(-\left(|0.02 \tau|^{2}\right)\right)$ \\
\hline$z_{1}$ & Normal & 0.8185 & 0.02701 & - \\
\hline$z_{2}$ & Normal & 0.9233 & 0.03047 & - \\
\hline$z_{3}$ & Normal & 1.065 & 0.03515 & - \\
\hline$z_{4}$ & Normal & 1.014 & 0.03346 & - \\
\hline$z_{5}$ & Normal & 1 & 0.033 & - \\
\hline$z_{6}$ & Normal & 1 & 0.033 & - \\
\hline$z_{7}$ & Normal & 1 & 0.033 & - \\
\hline$z_{8}$ & Normal & 2.468 & 0.01234 & - \\
\hline$z_{9}$ & Normal & 189.8 & 9.49 & - \\
\hline$z_{10}$ & Normal & 0.7524 & 0.003762 & - \\
\hline$z_{11}$ & Normal & 0.9935 & 0.0049675 & - \\
\hline$z_{12} / \mathrm{mm}$ & Normal & 140 & 0.7 & - \\
\hline$z_{13} / \mathrm{mm}$ & Normal & 191.489 & 0.9574 & - \\
\hline$z_{14}$ & Normal & 1.375 & 0.04538 & - \\
\hline$z_{15}$ & Normal & 1.156 & 0.03815 & - \\
\hline$z_{16}$ & Normal & 1.307 & 0.04313 & - \\
\hline$z_{17}$ & Normal & 1.0469 & 0.03455 & - \\
\hline
\end{tabular}

Note: $\tau$ is the time interval

Table 2 Failure probabilities at different time instances

\begin{tabular}{llll}
\hline $\begin{array}{l}\text { Service time } \\
t_{i_{\mathrm{t}}} / \mathrm{a}\end{array}$ & $\begin{array}{l}\text { MCS } P_{\mathrm{F}, i_{\mathrm{t}}} \\
\left(\times 10^{-3}\right)\end{array}$ & $\begin{array}{l}\text { FORM } P_{\mathrm{F}, i_{\mathrm{t}}} \\
\left(\times 10^{-3}\right)\end{array}$ & $\begin{array}{l}\text { SS } P_{\mathrm{F}, i_{\mathrm{t}}} \\
\left(\times 10^{-3}\right)\end{array}$ \\
\hline 1 & 1.56 & 1.56 & 1.53 \\
2 & 2.66 & 2.50 & 2.66 \\
3 & 3.32 & 3.28 & 3.34 \\
4 & 3.68 & 3.98 & 3.83 \\
5 & 4.52 & 4.62 & 4.49 \\
6 & 5.30 & 5.22 & 5.36 \\
7 & 5.46 & 5.78 & 5.52 \\
8 & 6.04 & 6.31 & 6.04 \\
9 & 6.74 & 6.82 & 6.74 \\
10 & 7.72 & 7.31 & 7.83 \\
\hline
\end{tabular}

computational times $t_{\mathrm{s}}$ acquired by the proposed method and MCS. For the same computing environment, Fig. 3 presents the variation in the COVs with an increasing failure probability. Furthermore, all values of $\delta_{i_{\mathrm{t}}}$ meet the condition of being less than $30 \%$ to provide adequate accuracy. Based on these conditions, compared to the computational time required for MCS, the time required for the proposed method is much shorter, as shown in Fig. 4. Consider the service time instance of $t=3$ a as an example. The number of samples required for MCS was $10^{6}$, but the proposed method required only $10^{3}$ initial samples. The computational time required by the proposed method $\left(t_{\mathrm{s}-}\right.$ $=6.9201 \mathrm{~s}$ ) is only $3.64 \%$ of the time required for the direct MCS $\left(t_{\mathrm{s}}=190.1115 \mathrm{~s}\right)$. These results demonstrate that the proposed method for estimating the failure probabilities of time-variant reliability for a high-dimensional continuous system with strength deterioration is relatively accurate and efficient.

\subsection{Tubular cantilever structure}

The tubular cantilever structure presented in Fig. 5 was considered as a second example. This example was studied by Madsen et al. [38], and Du and Chen [39] as an engineering design problem. The cantilever structure was analyzed based on its resistance to yielding caused by bending and sheering stress. Seven variables were involved in this example: two stochastic process variables $F_{1}(t)$ and $T(t)$, and five random variables $S_{0}, F_{2}, F_{3}, d$, and $h . S_{0}$ was the initial strength of the tubular cantilever structure and strength deterioration was modeled as a gamma process. $F_{1}(t), F_{2}, F_{3}$, and $T(t)$ are the three external forces and torque imposed on the cantilever structure, respectively, and $d$ and $h$ are the dimensions of the cross section. Their statistical distributions and parameters are listed in Table 3 and are all independent. Additionally, the locations of the force points and angles relative to vertical of the forces 


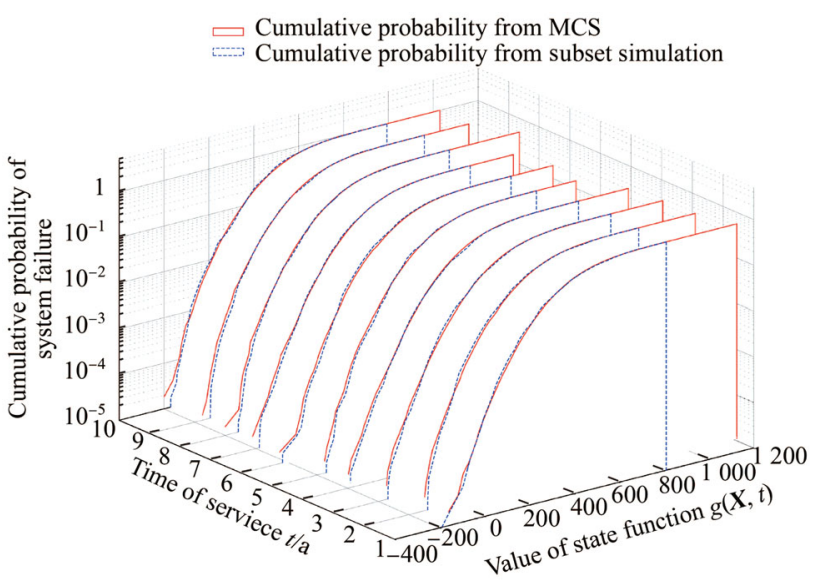

Fig. 2 Cumulative PDF curves of performance functions for a highdimensional continuous system

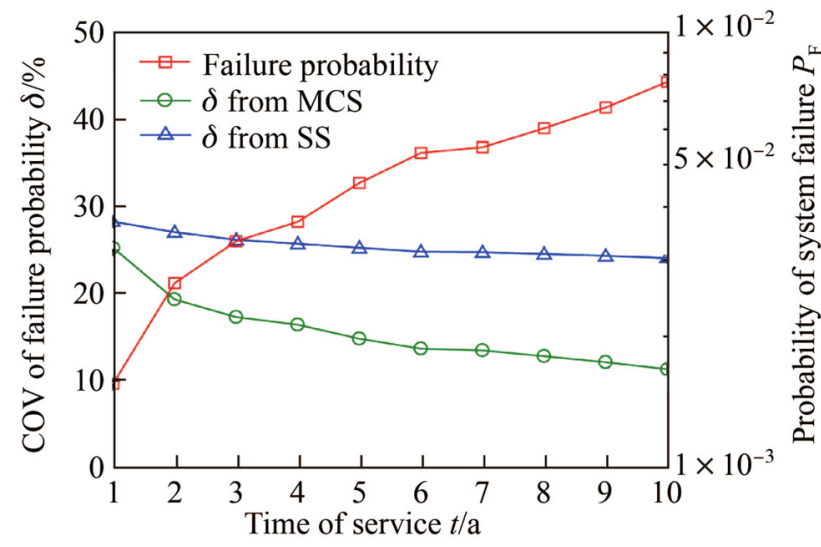

Fig. 3 Variations in the failure probabilities and COVs of a highdimensional continuous system

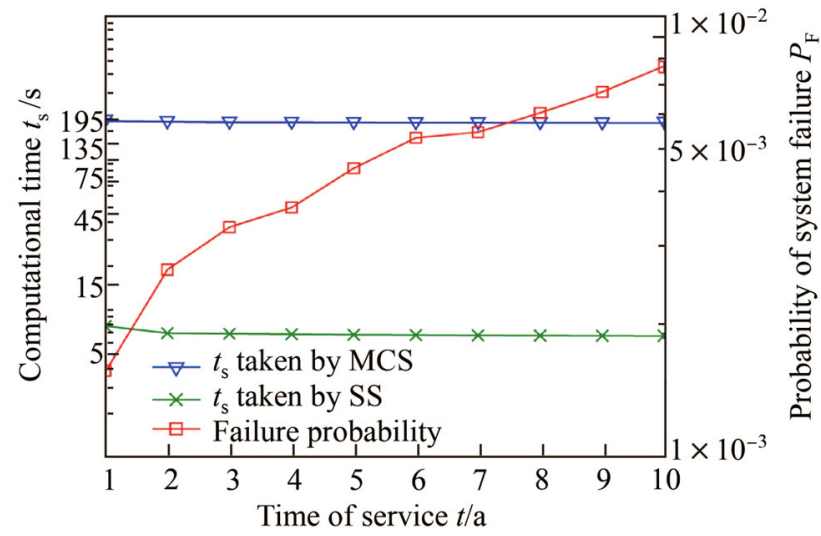

Fig. 4 Variations in the failure probabilities and computational times for a high-dimensional continuous system

$F_{1}(t)$ and $F_{2}$ were $L_{1}=60 \mathrm{~mm}, L_{2}=120 \mathrm{~mm}, \theta_{1}=5^{\circ}$, and $\theta_{2}=10^{\circ}$.

As shown in Fig. 5, $F_{1}(t), F_{2}, F_{3}$, and $T(t)$ cause the cantilever structure to experience bending and torsion.

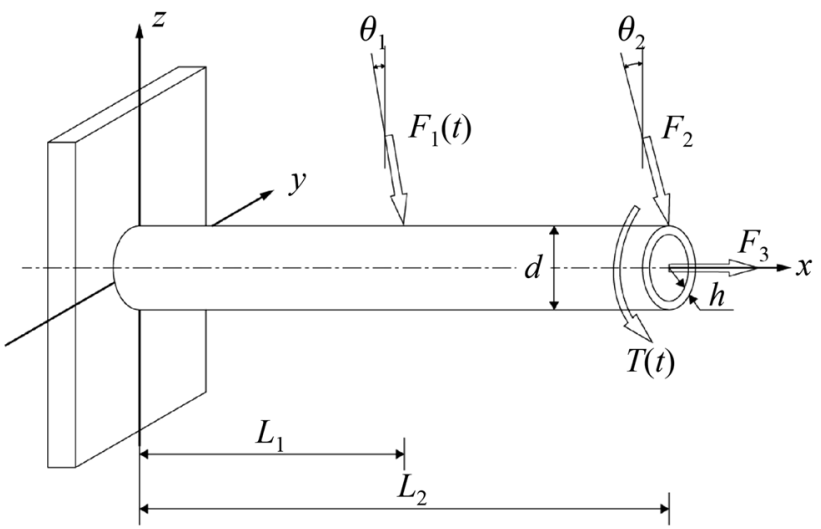

Fig. 5 Geometry of the tubular cantilever structure example

Based on analysis of mechanical model, the bending and sheering stresses can be calculated as follows

$$
\begin{aligned}
\sigma_{x}(t)= & \frac{F_{3}+F_{2} \sin \theta_{1}+F_{1}(t) \sin \theta_{2}}{\frac{\pi}{4}\left(d^{2}-(d-2 h)^{2}\right)} \\
& +\frac{\frac{d}{2}\left(F_{2} L_{1} \cos \theta_{1}+F_{1}(t) L_{2} \cos \theta_{2}\right)}{\frac{\pi}{64}\left(d^{4}-(d-2 h)^{4}\right)}, \\
\tau_{x z}(t)= & \frac{T(t) d}{4 \frac{\pi}{64}\left(d^{4}-(d-2 h)^{4}\right)} .
\end{aligned}
$$

According to Eqs. (14) and (15), the maximum stress can be expressed as

$$
\sigma_{\max }(t)=\sqrt{\sigma_{x}^{2}(t)+3 \tau_{x z}^{2}(t)} .
$$

Based on the deterioration of strength, the performance function takes on the following form

$$
\begin{aligned}
g(\boldsymbol{X}, t) & =S(t)-\max (\sigma(\boldsymbol{Z}, t)) \\
& =S_{0}-Y(t)-\sigma_{\max }(t),
\end{aligned}
$$

where $Y(t)$ is defined by parameters $u=1.4863 \times 10^{-6}$, $b=0.2$, and $c=2.8349 \times 10^{7}$.

The failure probability results are listed in Table 4. These results were obtained by the proposed method, FORM, and MCS based on Eq.(17). A comparison of the cumulative probability distributions of the tubular cantilever structure failure system as calculated by the proposed method and MCS at each service time instance is presented in Fig. 6. Figures 7 and 8 indicate that the computational time required by the proposed method is still much shorter than that required by MCS in the case of $\delta_{i_{\mathrm{t}}}<30 \%$.

For the instance of $t=1$, the number of samples required for MCS was $10^{6}$, but the proposed method required only $10^{3}$ initial samples. The computational time required by the proposed method $\left(t_{\mathrm{s}}=6.9519 \mathrm{~s}\right)$ is 
Table 3 Probability distributions of the basic random variables

\begin{tabular}{llrll}
\hline Random variable & Distribution & Mean & Standard deviation & Autocorrelation coefficient function \\
\hline$S_{0} / \mathrm{MPa}$ & Normal & 560 & 56 & - \\
$F_{1}(t) / \mathrm{N}$ & Gaussian process & 1800 & 180 & $\sin (|0.3 \tau|) /|0.3 \tau|$ \\
$F_{2} / \mathrm{N}$ & Normal & 1800 & 180 & - \\
$F_{3} / \mathrm{N}$ & Gumbel & 1000 & 100 & - \\
$T(t) /(\mathrm{N} \cdot \mathrm{mm})^{-1}$ & Gaussian process & 420000 & 42000 & $\exp (-|0.1 \tau|)$ \\
$d / \mathrm{mm}$ & Normal & 42 & 4.2 & - \\
$h / \mathrm{mm}$ & Normal & 5 & 0.5 & - \\
\hline
\end{tabular}

Note: $\tau$ is the time interval

Table 4 Failure probabilities at each time instance

\begin{tabular}{llll}
\hline $\begin{array}{l}\text { Service time } \\
t_{i_{\mathrm{t}}} / \mathrm{a}\end{array}$ & $\begin{array}{l}\text { MCS } P_{\mathrm{F}, i_{\mathrm{t}}} \\
\left(\times 10^{-3}\right)\end{array}$ & $\begin{array}{l}\text { FORM } P_{\mathrm{F}, i_{\mathrm{t}}} \\
\left(\times 10^{-3}\right)\end{array}$ & $\begin{array}{l}\text { SS } P_{\mathrm{F}, i_{\mathrm{t}}} \\
\left(\times 10^{-3}\right)\end{array}$ \\
\hline 1 & 0.38 & 0.261 & 0.381 \\
2 & 0.80 & 0.561 & 0.700 \\
3 & 1.16 & 0.947 & 1.220 \\
4 & 1.84 & 1.420 & 1.820 \\
5 & 2.58 & 1.990 & 2.580 \\
6 & 3.56 & 2.670 & 3.780 \\
7 & 4.26 & 3.440 & 4.540 \\
8 & 5.84 & 4.320 & 6.030 \\
9 & 7.02 & 5.310 & 7.230 \\
10 & 7.72 & 6.410 & 7.610 \\
\hline
\end{tabular}

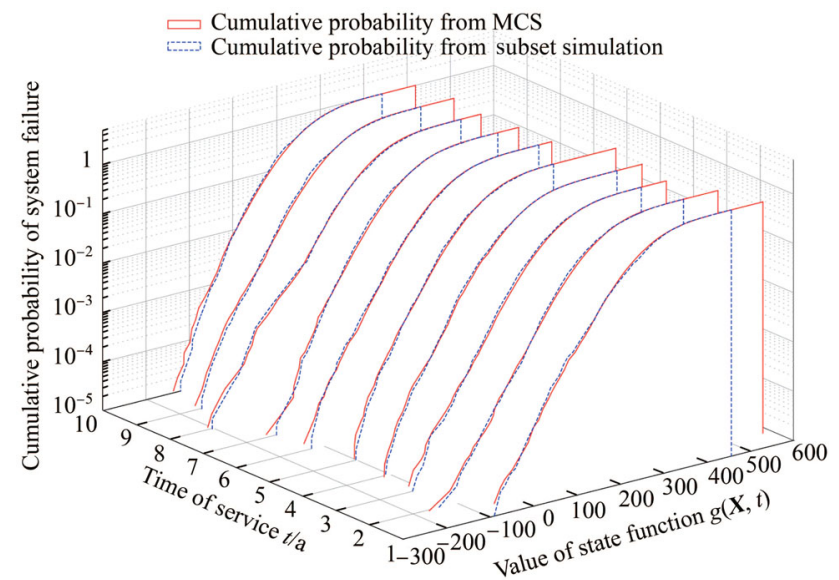

Fig. 6 Cumulative PDF curves of the performance function for the tubular cantilever structure

significantly shorter than that required for MCS $\left(t_{\mathrm{s}}=338.2885 \mathrm{~s}\right)$. And the $P_{\mathrm{F}, 1}$ value estimated by the proposed method is $3.81 \times 10^{-4}$, which agrees well with the result obtained from MCS, $3.80 \times 10^{-4}$. However, the $P_{\mathrm{F}, 1}$ value calculated by the FORM is $9.87 \times 10^{-6}$, which differs significantly from the MCS result. This discrepancy

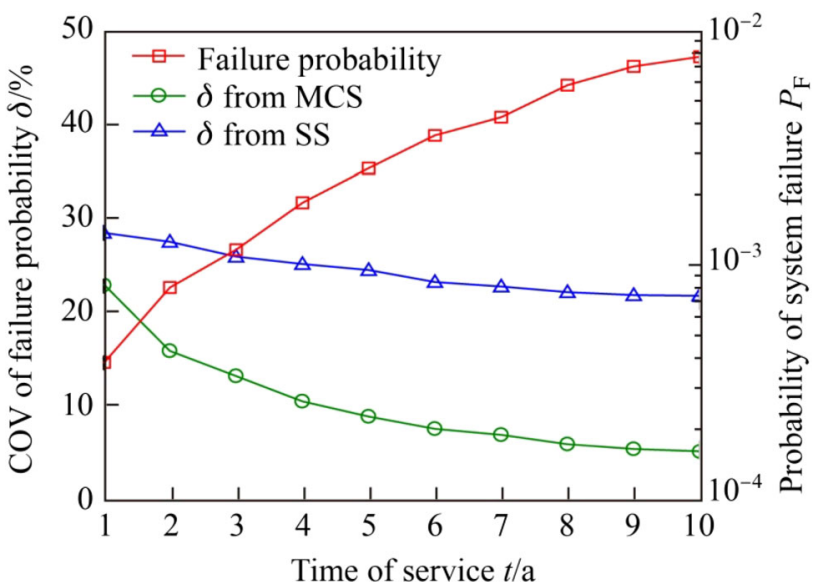

Fig. 7 Variations in the failure probabilities and COVs for the tubular cantilever structure

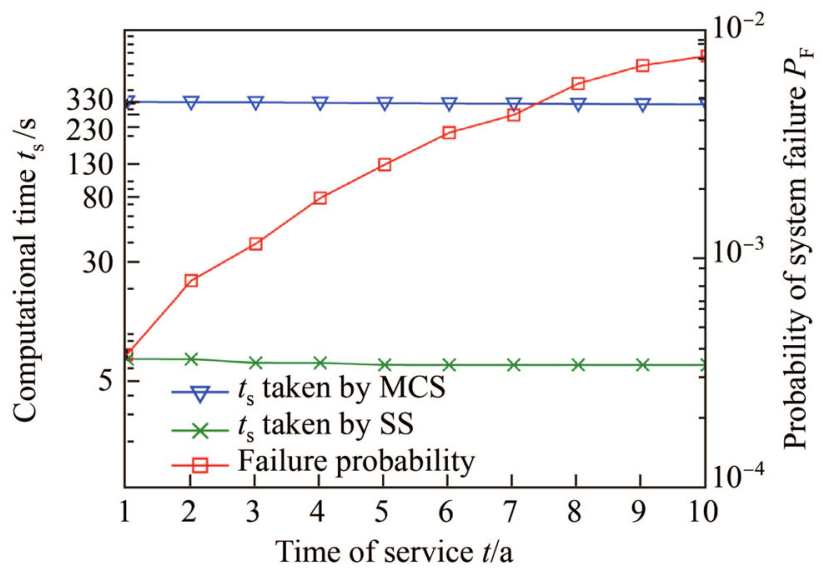

Fig. 8 Variations in the failure probabilities and computational times for the tubular cantilever structure

is largely based on the errors induced by the nonlinearity of the performance function for the tubular cantilever structure system and variety of the distributions of random variables. Additionally, the failure probabilities of the events were all small. These results further demonstrate that SS modified for a continuous system is an accurate and 
efficient method for estimating time-variant reliability in engineering design problems with strength deterioration and small failure probabilities.

\section{Conclusions}

A novel reliability analysis method was proposed in this paper. The proposed method combines the advantages of SS with the discretization of continuous systems. Continuous service times are divided into appropriate time intervals to determine the variation in failure probabilities for continuous systems at each time instance using SS. This method evaluates the small failure probabilities of timevariant reliability with strength deterioration efficiently and accurately. The proposed model for time-variant reliability with strength deterioration performs well on reliability analysis problems for continuous systems subjected to strength deterioration and dynamic loads.

Two examples demonstrated that the proposed method estimates $P_{\mathrm{F}, i_{\mathrm{t}}}$ values reasonably accurately compared to common reliability analysis methods (e.g., the FORM), particularly if the failure probabilities are as low as $10^{-4}$. Furthermore, the computational time required for the proposed method is considerably shorter than that required for MCS. The proposed method can be utilized for continuous systems with small failure probabilities as an effective reliability analysis method. Additionally, it provides a new perspective for time-variant reliability problems with strength deterioration characterized by small failure probabilities, multiple random variables, and nonlinearity of performance functions.

Acknowledgements The authors gratefully acknowledge the support of the National Natural Science Foundation of China (Grant Nos. U1708254 and 51575094).

Open Access This article is distributed under the terms of the Creative Commons Attribution 4.0 International License (http://crea tivecommons.org/licenses/by/4.0/), which permits unrestricted use, distribution, and reproduction in any medium, provided you give appropriate credit to the original author(s) and the source, provide a link to the Creative Commons license, and indicate if changes were made.

\section{References}

1. Castaldo P, Palazzo B, Mariniello A (2017) Effects of the axial force eccentricity on the time-variant structural reliability of agingcross-sections subjected to chloride-induced corrosion. Eng Struct 130:261-274

2. Huang X, Li Y, Zhang Y et al (2018) A new direct second-order reliability analysis method. Appl Math Model 55:68-80

3. Zhu SP, Huang HZ, Peng WW et al (2016) Probabilistic physics of failure-based framework for fatigue life prediction of aircraft gas turbine discs under uncertainty. Reliab Eng Syst Saf 146:1-12

4. Mori Y, Ellingwood BR (1993) Reliability-based service-life assessment of aging concrete structures. J Struct Eng 119(5):1600-1621

5. Li CQ (1995) Computation of the failure probability of deteriorating structural systems. Comput Struct 56(6):1073-1079

6. Ciampoli M (1998) Time dependent reliability of structural systems subject to deterioration. Comput Struct 67(1-3):29-35

7. Li Q, Wang C, Ellingwood BR (2015) Time-dependent reliability of aging structures in the presence of non-stationary loads and degradation. Struct Saf 52:132-141

8. Rice SO (1944) Mathematical analysis of random noise. Bell Syst Tech J 23(3):282-332

9. Andrieu-Renaud C, Sudret B, Lemaire M (2004) The PHI2 method: a way to compute time-variant reliability. Reliab Eng Syst Saf 84(1):75-86

10. Zhang XJ, Xie LY, Wu Y et al (2010) Modeling for time-variant reliability of mechanism. Adv Mater Res 118-120:621-624

11. Li CC, Kiureghian AD (1993) Optimal discretization of random fields. J Eng Mech 119(6):1136-1154

12. Au SK, Beck JL (2001) Estimation of small failure probabilities in high dimensions by subset simulation. Probab Eng Mech 16(4):263-277

13. Au SK, Beck JL (2003) Subset simulation and its application to seismic risk based on dynamic analysis. J Eng Mech 129(8):901-917

14. Vahdatirad MJ, Andersen LV, Ibsen LB et al (2014) Stochastic dynamic stiffness of a surface footing for offshore wind turbines: implementing a subset simulation method to estimate rare events. Soil Dyn Earthq Eng 65:89-101

15. Norouzi M, Nikolaidis E (2013) Integrating subset simulation with probabilistic re-analysis to estimate reliability of dynamic systems. Struct Multidiscip Optim 48(3):533-548

16. Song SF, Lu ZZ, Qiao HW (2009) Subset simulation for structural reliability sensitivity analysis. Reliab Eng Syst Saf 94(2):658-665

17. Bourinet JM, Deheeger F, Lemaire M (2011) Assessing small failure probabilities by combined subset simulation and support vector machines. Struct Saf 33(6):343-353

18. Zuev KM, Beck JL, Au SK et al (2012) Bayesian post-processor and other enhancements of subset simulation for estimating failure probabilities in high dimensions. Comput Struct 92-93:283-296

19. Li HS, Ma YZ, Cao ZJ (2015) A generalized subset simulation approach for estimating small failure probabilities of multiple stochastic responses. Comput Struct 153:239-251

20. Wang Z, Mourelatos ZP, Li J et al (2014) Time-dependent reliability of dynamic systems using subset simulation with splitting over a series of correlated time intervals. J Mech Des 136(6):061008

21. Yu S, Wang ZL (2018) A novel time-variant reliability analysis method based on failure processes decomposition for dynamic uncertain structures. J Mech Des 140(5):051401

22. Yu S, Wang ZL, Meng DB (2018) Time-variant reliability assessment for multiple failure modes and temporal parameters. Struct Multidiscip Optim 58(4):1705-1717

23. Abdel-Hameed M (1975) A gamma wear process. IEEE Trans Reliab 24(2):152-153

24. Van Noortwijk JM (2009) A survey of the application of gamma processes in maintenance. Reliab Eng Syst Saf 94(1):2-21

25. Ellingwood BR, Mori Y (1993) Probabilistic methods for condition assessment and life prediction of concrete structures in nuclear power plants. Nucl Eng Des 142(2-3):155-166

26. Cinlar E, Osman E, Bazant ZP (1977) Stochastic process for extrapolating concrete creep. J Eng Mech Div 103(6):1069-1088 
27. Hoffmans GJCM, Pilarczyk KW (1995) Local scour downstream of hydraulic structures. J Hydraul Eng 121(4):326-340

28. Van Noortwijk JM, Klatter HE (1999) Optimal inspection decisions for the block mats of the eastern-scheldt barrier. Reliab Eng Syst Saf 65:203-211

29. Papaioannou I, Betz W, Zwirglmaier K et al (2015) MCMC algorithms for subset simulation. Probab Eng Mech 41:89-103

30. Metropolis N, Rosenbluth AW, Rosenbluth MN et al (1953) Equation of state calculations by fast computing machines. J Chem Phys 21:1087-1092

31. Zhao YG, Ono T (1999) A general procedure for first/secondorder reliability method (FORM/SORM). Struct Safe 21(2):95-112

32. Baumgärtner A, Binder K (1987) Applications of the Monte Carlo method in statistical physics. Springer, Berlin

33. Zhang YM, He XD, Liu QL et al (2005) Robust reliability design of banjo flange with arbitrary distribution parameters. J Press Vessel Technol 127(4):408-413
34. O'Connor AN (2011) Probability distributions used in reliability engineering. University of Maryland, Maryland

35. Bellman RE (1961) Adaptive control processes: a guided tour. Princeton University Press, New Jersey

36. International Organization for Standards (2006) ISO 6336-2-2006 calculation of load capacity of sour and helical gears-part 2: calculation of surface durability (pittings). International Organization for Standards, Switzerland

37. Au SK, Wang Y (2014) Engineering risk assessment with subset simulation. Wiley/Blackwell, New Jersey

38. Madsen HO, Krenk S, Lind N (1986) Methods of structural safety. Prentice Hall, New Jersey

39. Du XP, Chen W (1999) Towards a better understanding of modeling feasibility robustness in engineering design. J Mech Des 122(4):385-394 